\title{
The Strength beyond the Muscle
}

\author{
Karen T. Nozoe, Gustavo A. Moreira, MD, PhD, Helena Hachul, MD, PhD, \\ Sergio Tufik, MD, PhD, and Monica L. Andersen, PhD
}

$\mathbf{F}$ ATAL OR CHRONIC DISEASES AFFLICT both body and soul, causing the sick person to feel as if he or she were drifting in a boat with no destination. However, in some cases, especially in which the afflicted patient is a child, a companion such as the child's mother shares the boat as it sails. Then, it is she who steers this vessel, and often alone at the helm, she sails for days on end. On her course there are storms and darkness and no map or guidance. Still, due to that single passenger-her child-she finds a light that shows her the way and warms her heart. Many times she feels her strength wane, and she might suddenly lose her grip and capsize. There are, however, places along the way where she may harbor and sojourn, and our center is one such place; we will join these companions for a while on their boats and try to provide them with light and fuel so that they may travel further.

Day after day, with each new report from a mothercaregiver, we learn to appreciate even more the relationship between mother and child and the unconditional maternal love. The interaction between a doctor/researcher, the patient/volunteer, and his or her family extends beyond preestablished protocols. Each one of these individuals brings many stories, expectations, hopes, and dreams that are placed in the specialists' hands, transcending the restricted professional barrier. In this scenario, we would like to share our experience not only with a specific individual, but with a unique group of women: the mother-caregivers of children affected by Duchenne Muscular Dystrophy (DMD).

DMD is a hereditary neuromuscular disorder characterized by progressive muscle weakness and reduced muscle tone, mainly affecting men. This disease heavily impacts affected individuals, significantly compromising their health, quality of life, and especially their freedom. DMD patients manifest the disease in childhood, with locomotor and respiratory impairments which will lead to an early death without appropriate medical care. Unfortunately, until now there is no cure for this disease. Our group has a specialized clinic (TDN), accredited by the Brazilian public health system to provide medical and physiotherapy treatment for patients with neuromuscular disease who develop respiratory including DMD. This center was conceptualized by a man who, besides performing as a doctor and researcher, was determined to help this group of patients. Dissatisfied with the state of care, the doctor and mastermind of this project fought not only for the acquisition and supply of NIV equipment, but also for the quality of life of DMD patients. After several attempts, he finally opened the TDN, which provided NIV to all DMD patients supported by this center. As a result, lives were prolonged, because affected children who usually died in their youth now become adults, with a high quality of life and the ability to achieve many of their dreams.

Furthermore, the observed benefit was not only to the patient. The family could also benefit from these achievements, and from among the family, the mother stands out. We emphasize the role of the mother, who assumes the role of caregiver, giving up her professional and personal life to take care of and stand beside her son.

Bravely, the mother-caregiver fights to achieve a life with better quality and expectations for her son, seeking good doctors in the area, the best available treatment, including the acquisition of the NIV, and incessantly searching for more and new information about the disease. Thus, many mothercaregivers come to our center with their children, bringing lots of strength and hope. We are spectators for many stories of the determination and struggle of these women. When this caretaking mother alights at our center, we notice the eager look in her eyes, like that of a child who, after having long waited for a gift, anxiously and hurriedly tears through the wrapping paper of the beautiful, colorful package to find what's inside. It is then, with this look in her eyes, with the hope to learn fresh and surprising news regarding her child's disease, that she asks, "Any new developments in the treatment for Duchenne Muscular Dystrophy?" We feel torn, as we must tell her that, so far, there is no concrete treatment or cure for this disease. Facing the empty look of disappointment in the mother's eyes, opening the package only to find it empty, we grope in our own confusion for words and manage to say, "Do keep up faith and hope; research is progressing." During this moment, we also heard the following story from a mother: "My son asked me to watch a movie in the cinema, but upon arriving at the place there was no way to enter with him in the wheelchair. When I saw the sadness and disappointment in my son's eyes, I took him in my arms with all my strength and carried him to his place at the cinema. It was very gratifying to see him watching that movie, happy, but at the same time I felt sad to have to go through this with my son. It was painful to see my son wanting something simple that became difficult because of his condition, and it makes me think several times before taking him somewhere nowadays." In another case, it was observed in the clinic that the

Department of Psychobiology, Universidade Federal de São Paulo, São Paulo, Brazil. 
patient had a compromised nutritional status and weight loss due to the worsening of the disease. When a gastrostomy was suggested, to prevent possible malnutrition, tears flowed from the eyes of the mother-caregiver: "My son told me he was wanting to eat lasagna. And now, how will he eat? Will he still be able to taste the food? Can I still prepare the meals he likes so much?"

On the other hand, due to the good prognosis because of the introduction of the NIV, we have heard patients reporting their dreams of going to college and conquering the job market. Always by his side, the patient's mother smiles, happy and proud, ready to fight with her child for these dreams. We emphasize in this report the strength of this mother-caregiver, a warrior woman that is also gentle and loving. These mothers are always ready to take care of their children, with no hesitation.

But what about them? What about their health and wellbeing? Their feelings? Who thinks about them and takes care of these brave mother-caregivers? In this scenario, precisely because of these concerns and all the described reports, and due to our admiration for their unique courage, this experience already takes place at the TDN and especially in the lives of all involved professionals. This life experience leads us to always search for the best for the patient, and guides our attention to the health and well-being of the mother-caregiver, so that we can support this woman who helps us so much, since a mother understands even what a child does not say. We cannot save those children and mothers, but we can reach out to them and provide them with a harbor where they can moor, rest and recharge to carry on with their long journey.

\section{Acknowledgments}

We would like to acknowledge the invaluable support and help of Laís Berro and Daniel Polesel. Neither of the authors have any conflict of interest to disclose. Our studies are supported by grants from Associação Fundo de Incentivo à Pesquisa (AFIP) and São Paulo Research Foundation (FAPESP) (grant \#2012/08587-8 to K.T.N.). S.T. and M.L.A. received $\mathrm{CNPq}$ fellowships.

Address correspondence to:

Monica L. Andersen, PhD

Department of Psychobiology Universidade Federal de São Paulo São Paulo, Brazil

E-mail: mandersen@unifesp.br 